\title{
Mbd3 Promotes Reprogramming of Primary Human Fibroblasts
}

\author{
Sajjida Jaffer ${ }^{1,2}$, Pollyanna $\mathrm{Goh}^{4}$, Mahnaz Abbasian ${ }^{1,2}$, Amit C Nathwani ${ }^{1,2,3}$ \\ ${ }^{I}$ Department of Haematology, University College London, Cancer Institute, London, UK \\ ${ }^{2}$ Katharine Dormandy Haemophilia and Thrombosis Centre, Royal Free NHS Trust, London, UK \\ ${ }^{3}$ National Health Services Blood and Transplant, Oak House, Reeds Crescent, Watford, Hertfordshire, UK \\ ${ }^{4}$ Centre for Paediatrics, Barts and The London Medical School, Blizard Institute, Queen Mary University of London, London, UK
}

Mbd3 (Methyl-CpG binding domain protein), a core member of NuRD (nucleosome remodelling and deacetylation) is essential for embryogenesis. However, its role in reprogramming of somatic cells into induced pluripotent stem cells (iPSC) remains controversial. Some reports suggest that Mbd3 inhibits pluripotency, whilst others show that it greatly enhances reprogramming efficiency. Our study is the first to assess the role of $M b d 3$ on reprogramming of primary human fibroblasts using Yamanaka episomal plasmids (Reprogramming factors (RF) under feeder-free conditions. We showed that shRNA-mediated partial depletion of $M b d 3$ resulted in $>5$-fold reduction in the efficiency of reprogramming of primary human fibroblasts. Furthermore, iPSC that emerged after knock-down of Mbd3 were incapable of trilineage differentiation even though they expressed all markers of pluripotency. In contrast, over-expression of the $M b d 3 b$ isoform along with the Yamanaka episomal plasmids increased the number of fibroblast derived iPSC colonies by at least two-fold. The resulting colonies were capable of trilineage differentiation. Our results, therefore, suggest that $M b d 3$ appears to play an important role in reprogramming of primary human fibroblasts, which provides further insight into the biology of reprogramming but also has direct implication for translation of iPSC to clinic.

Keywords: Mbd3, Induced pluripotent stem cells, Episomal reprogramming, Fibroblasts

\section{Introduction}

The pioneering work by Takahashi and Yamanaka showed that induced pluripotent stem (iPSC) cells can be generated from somatic cells by ectopic expression of four different transcription factors, originally Oct4, Sox2, Klf4 and $M y c$ (1-3). Understanding the molecular mechanisms

Received: April 23, 2018, Revised: April 23, 2018,

Accepted: September 25, 2018, Published online: November 30, 2018 Correspondence to Amit C Nathwani

Department of Haematology, University College London, Cancer Institute, 72 Huntley Street, London, WC1E 6DD, UK

Tel: +44-(0)20-7794-0500, Fax: +44-(0)20-7472-6759

E-mail: amit.nathwani@ucl.ac.uk

(c) This is an open-access article distributed under the terms of the Creative Commons Attribution Non-Commercial License (http://creativecommons.org/ licenses/by-nc/4.0/), which permits unrestricted non-commercial use, distribution, and reproduction in any medium, provided the original work is properly cited.

Copyright (C) 2018 by the Korean Society for Stem Cell Research that regulate the pluripotency and self-renewal of iPSC is critical for regenerative medicine in generating target cells for transplantation. Reprogramming requires major epigenetic modifications of DNA and chromatin remodelling and several groups are investigating the role of Nucleosome Remodelling and Histone Deacetylase (NuRD) complex and in particular Mbd3 (Methyl-CpG binding domain protein). $M b d 3$ is ubiquitously expressed in all somatic cells and is required for formation of stable NuRD complex (4). Deletion of the $M b d 3$ gene causes embryonic lethality in mice. Additionally, embryonic stem cells lacking $M b d 3$ protein fail to commit to developmental lineages (4, 5). Whilst it is clear that $M b d 3$ is required for early embryonic development and stem cell fate decisions, its exact role in somatic cell reprogramming is currently unclear. Two reports suggest that $M b d 3$ inhibits pluripotency as its reduction or deletion leads to an enhanced kinetics of reprogramming with iPSC colonies appearing within 7 days and with an almost $100 \%$ reprogramming efficiency $(6,7)$. 
In contrast, a study by dos Santos et al. (8), showed that loss of $M b d 3$ expression, especially, during the initiation phase of reprogramming leads to a reduction in the efficiency of reprogramming. It is unclear if these disparate results reflect the different reprograming techniques used or a function of the target cells.

Here, we investigated the role of $M b d 3$ in direct reprogramming of primary human fibroblasts using episomal-plasmid based method $(2,3)$. Our results show that over-expression of $M b d 3 b$ significantly increased the yield of iPSC colonies and reduction of $M b d 3$ expression not only reduced the yield of iPSC colonies but repressed subsequent trilineage differentiation. Our findings suggest that $M b d 3$ plays a critical role in reprogramming of primary human fibroblasts at least in the context of episomal-based method of reprogramming.

\section{Materials and Methods}

\section{Cell culture}

All human primary fibroblast cells were generated in vitro after written informed consent using protocols approved by the Royal Free research ethics committee, Royal Free Hospital, London, UK. Fibroblasts were derived from a skin biopsy and cultured as described previously (3). We used PD1 (70 year old suffering from Parkinson disease) and RDP1 (49 year old with rapid onset Parkinsonism dystonia) as described previously (3). We also used BJ fibroblast cell line (a normal neonatal fibroblast cell line from ATCC).

We employed episomal plasmid method of reprogramming as described previously $(2,3)$ and used the following plasmids: pCXLE-hOct3/4-shp53 (Addgene number 27077), pCXLE-hSK (Addgene number 27078), pCXLEhUL (Addgene number 27080), (the latter three referred to as reprogramming factors- (RF)), and pCXLE-EGFP (Addgene number 27082). For inhibition of $\mathrm{Mbd3}$, we used pSMP-MBD3_1 (Addgene number 36371) (9). The $\mathrm{Mbd} 3$ isoform that we used for over-expression experiments was $M b d 3 b$ (8) which shares $>80 \%$ homology to human $M b d 3$ (10) and is the most abundant isoform in embryonic stem cells (11). The outline of experiments is summarized in Fig. 1A. Briefly, cells were transfected using program U-023 on a Nucleofector (I) $2 \mathrm{~b}$ device (Amaxa Nucleofector kit R) or Pulse code - EO150 (solution P2) on AMAXA 4D Nucleofector (LONZA). GFP transfection efficiency was measured using flow cytometry after a minimum of 24 hours of transfection. Established iPSC cell lines were maintained as described previously (3) and were passaged at $1: 1 \sim 1: 12$ using gentle cell dissociation reagent (12). At day 25 30 post transfection, cells were either fixed for whole plate alkaline phosphatase staining as per manufacturer's instructions or iPSC colonies were picked for expansion.

\section{Immunoblotting}

Immunoblotting was carried out as described previously (13). The blots were probed either with Mbd3 (ABCAM), $\alpha$-tubulin (ABCAM), or GAPDH (Cell Signalling). Densitometry was performed using NIH image J software and a sampling window was used to measure the intensity of all bands on the blot and normalized against the band intensity of the internal control.

\section{Characterisation of iPSC}

Immunofluorescence was carried on established iPSC cell lines as described previously (3). Primary antibodies namely TRA-1-60 and TRA-1-80 (Santa Cruz Biotechnologies) were used at a $1: 100$ dilution followed by goat anti-mouse $\operatorname{IgG} / \operatorname{IgM}$ Alexa 488 as a secondary antibody at a concentration of $1: 400$. The cells were visualised using Axiovision microscope equipped with $10 \times$ objective. For established iPSC cell lines, RNA was harvested with TRIzol and $1 \mu \mathrm{g}$ of RNA was used for subsequent reverse transcriptase reactions with Superscript III first strand synthesis system. For qRT-PCR cDNA was diluted in 1 in 20 and were set up in duplicates using SYBR Green Master Mix. Delta Ct values with GAPDH were calculated and brought to power -2 . Error bars represent \pm SEM of technical duplicates. Oligonucleotide primers details have been described elsewhere (3). To demonstrate spontaneous in vitro differentiation, iPSC were grown to confluency and maintained in suspension in EB media. The expression of trilineage marker genes were assayed after 14 days of differentiation (3). Statistical significance was determined by non-parametric Mann-Whitney U-test and in all cases $\mathrm{p} \leq 0.05$ was considered to be significant (Supplementary Methods).

\section{Results}

\section{Mbd3 is required for reprogramming of primary human fibroblasts}

In order to assess the role of $M b d 3$ in establishment of pluripotency of human somatic cells, a plasmid construct expressing short-hairpin (shp) RNA targeted to human $M b d 3$ transcript (shpMbd3) was transfected together with Yamanaka episomal plasmids expressing human Oct3/4, shRNA against p53, Sox2, Klf4 and L-Myc/Lin28 (referred to as $\mathrm{RF}$ ) in $\mathrm{BJ}$ foetal fibroblast cell line and two adult 
A

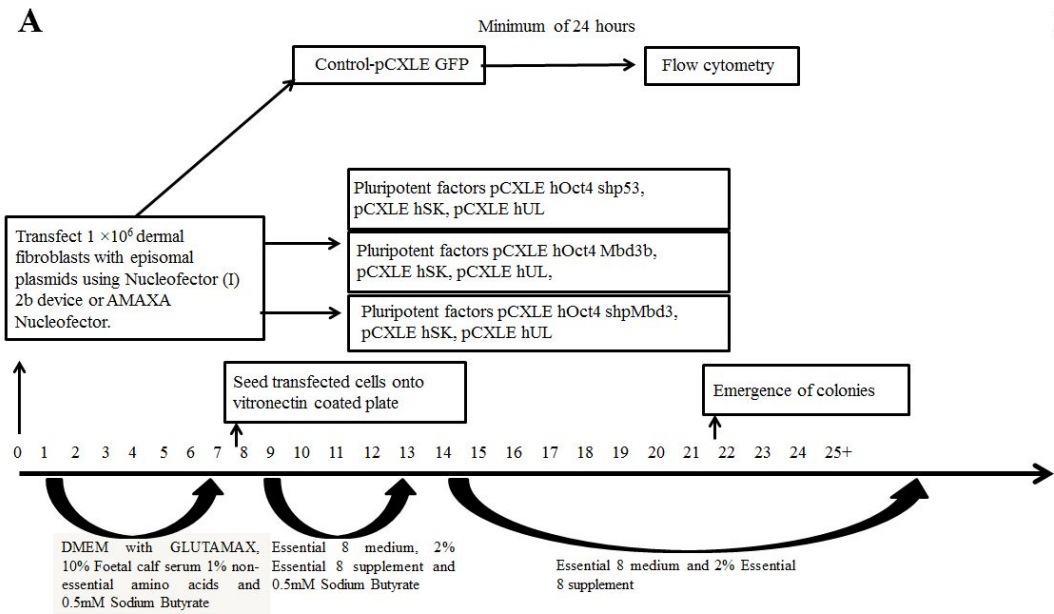
essential aminin acids

C

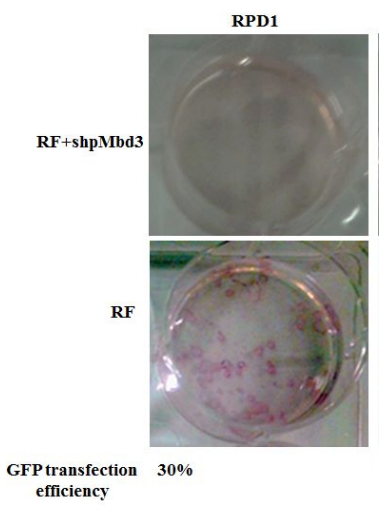

PD1

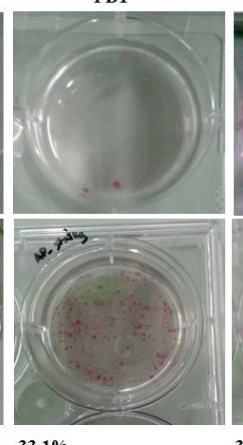

33.1\%

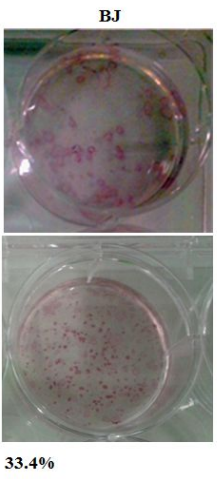

D
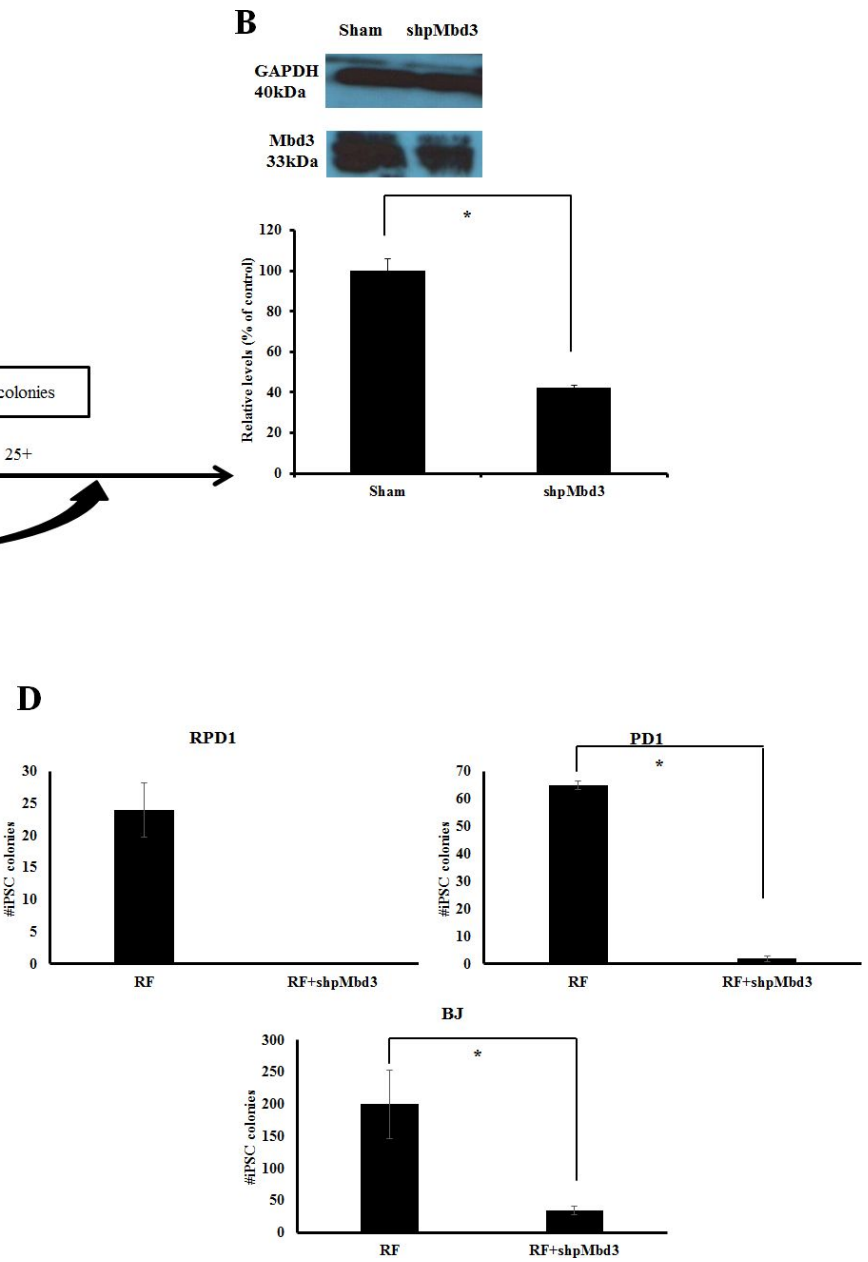

Fig. 1. Mbd3 is required for Episomal Reprogramming. (A) Experimental design used to address the kinetics and efficiency of reprogramming in human dermal fibroblasts. Human fibroblasts were transfected with episomal plasmids encoding pCXLE-hOct3/4-shp53, pCXLE-hSK (Sox-2 and Klf4) and pCXLE-hUL (L-Myc and Lin28) (Reprogramming factors-RF) or RF + shpMbd3 or RF + Mbd3b or GFP only. After transfection, the cells were maintained in Essential 8 media supplemented with $0.5 \mathrm{mM}$ sodium butyrate until day 12 and thereafter maintained in Essential 8 media only. Transfection efficiency was measured by Flow Cytometry after a minimum of 24 hours of transfection with an average of $32.8 \pm 1.68 \%$. (B) Immunoblot analysis for Mbd3 expression transfected with shpMbd3 compared to sham. Densitometry plot shows that cells transfected with shpMbd3 had 50\% reduction in expression of Mbd3 compared to sham $(p<0.05)$. (C) iPSC colony formation in fibroblasts was assessed by alkaline phosphatase (AP) staining between day $25 \sim 30$ and the results showed that shpMbd3 reduced the number of iPSC colonies. (D) Quantification of AP staining further confirmed reduction of iPSC colonies formed in cells treated with $\mathrm{RF}+\operatorname{shpMbd} 3$ compared with RF in patient fibroblasts RPD1 and PD1 and BJ neonatal cell line $(n=3)$. Colony number is per $1 \times 10^{6}$ fibroblasts $(p<0.05)$.

patient derived fibroblast lines; PD1 (70 years old with Parkinson's disease) and RDP1 (49 years old with rapid onset of Parkinsonism dystonia) (Fig. 1A). GFP transfection efficiency of all three lines was comparable with mean $( \pm S D)$ expression levels of $32.8 \pm 1.68 \%$. Immunoblotting after 24 48 hours of transfection showed 50\% knock-down of Mbd3 compared to sham $(\mathrm{p}<0.05)$ (Fig. 1B), but did not result in a difference in the proliferation rate of cells transfected with $\mathrm{RF}+\mathrm{shpMbd} 3$ when compared with cells transfected with RF alone as seen morphologically. This suggests that partial knock-down of
$M b d 3$ does not cause cell cycle arrest (8) of human fibroblasts, however, cell cycle analysis are required to further confirm this. iPSC colonies emerged at approximately the same time (days 12 20 post transfection) in the cells transfected with $\mathrm{RF}+$ shpMbd 3 and $\mathrm{RF}$ alone cultures suggesting that $M b d 3$ does not alter the kinetics of reprogramming as reported previously (8). We used alkaline phosphatase activity as an early indicator of pluripotency prior to performing an extended assessment of pluripotent stem cell markers (14). Our results showed that the number of alkaline phosphatase positive colonies in cells transfected 
with $R F+\operatorname{shpMbd} 3$ was significantly reduced $(\mathrm{p}<0.05)$ by 5 33 fold in established BJ fibroblast cell line and in PD1 and RPD1 primary fibroblast cells compared to the numbers observed with cells transfected with RF alone (BJ mean ( \pm SD): RF $200 \pm 52.9$ and $R F+\operatorname{shpMbd} 335 \pm 6.8$; RPD1 mean ( \pm SD): RF $24 \pm 4.2$ and $R F+\operatorname{shpMbd} 30 \pm 0$; PD1 mean $( \pm S D)$ : RF $65 \pm 1.5$ and $R F+\operatorname{shpMbd} 32 \pm 1$; $(n=3)$ Fig. 1C, D). Interestingly, this effect was more profound in patient derived fibroblasts than in neonatal $\mathrm{BJ}$ fibroblast cell-line. The reason for this difference is unclear but could reflect the biological age of these lines and disease status of the patients. It was however, possible to expand iPSC colonies from cells treated with RF+ shpMbd3 although less efficiently and with delayed kinetics compared to colonies expanded from cells treated
A

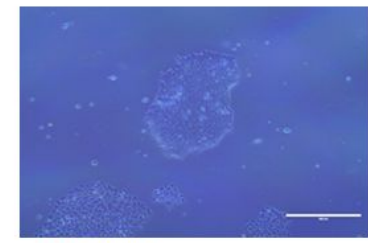

TRA-1-81

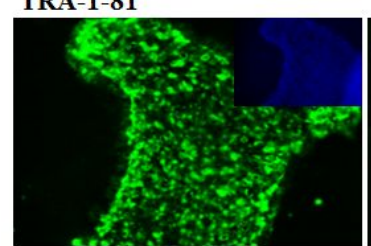

TRA-1-60
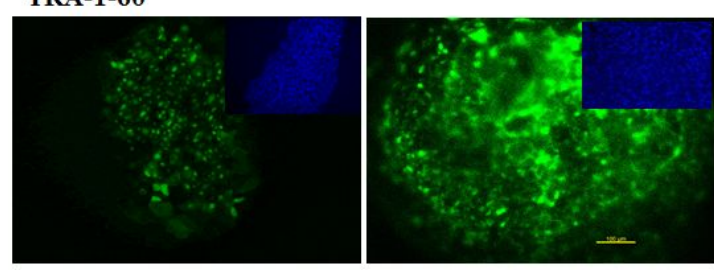

C

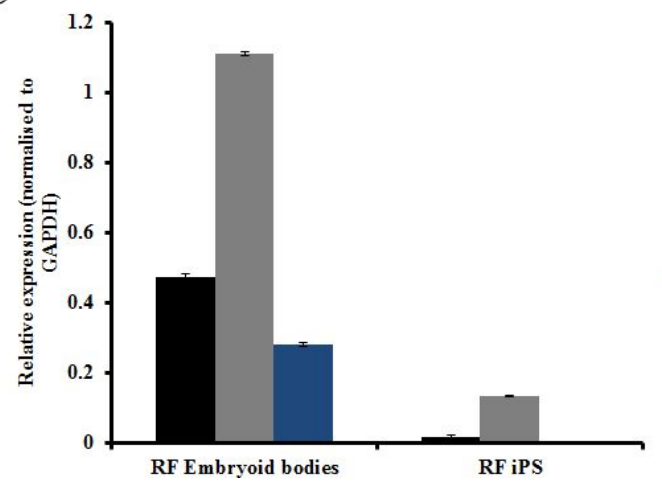

with RF only. Phenotypic characterisation of expanded iPSC colonies showed that they expressed TRA-1-60 and TRA-1-80 pluripotent markers with no difference in level of expression between cells treated with $\mathrm{RF}+\operatorname{shpMbd} 3$ and RF only (Fig. 2A). Additionally, qRT-PCR analysis showed that iPSCs expanded from RF and RF+shpMbd3 expressed pluripotent genes such as Oct-4 and Nanog (Fig. 2B) at comparable levels. To investigate the role of NuRD in differentiation, iPSCs were maintained in suspension in EB media and under these conditions iPSCs formed aggregates called embryoid bodies which consists of a heterogeneous mix of derivatives of the three germ layers. iPSCs derived from $\mathrm{RF}+\mathrm{shpMbd} 3$ failed to form EBs as previous reports (4). In contrast, EBs emerged from RF iPSCs with evidence of expression of trilineage markers
B

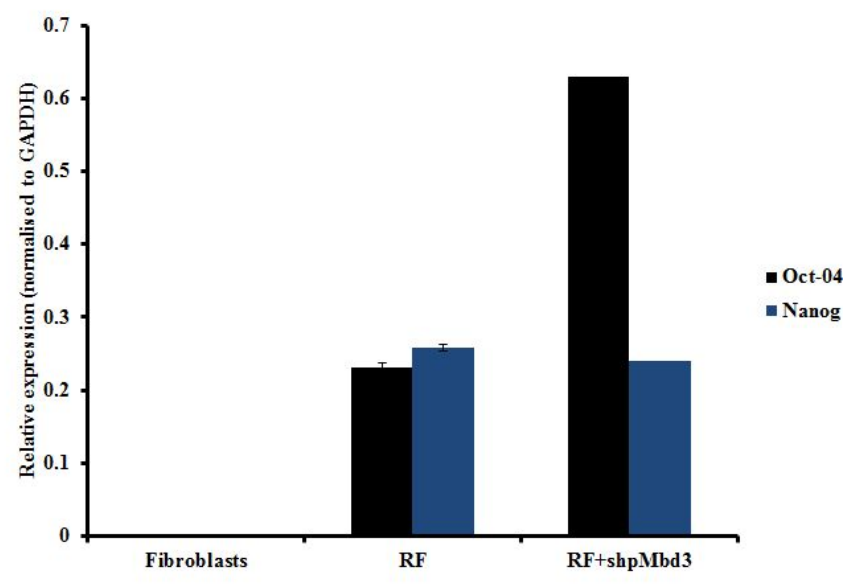

Fig. 2. Characterisation of iPSC colonies. (A) Representative phase images of iPSC derived from fibroblasts transfected RF and cells transfected with RF +shpMbd3. Morphologically they appear to be similar. Immunostaining with pluripotency markers (green) and counter staining with DAPI (blue) of iPSC derived from fibroblasts transfected with RF only and those transfected with $\mathrm{RF}+\operatorname{shpMbd} 3$ with TRA-1-81 and TRA-1-60. Scale bar is $200 \mu \mathrm{m}$. (B) Q-PCR showed expression of pluripotency genes (Oct-4 and Nanog respectively) in cells treated with RF and RF+ shpMbd3. All results were normalised to GAPDH. ( $n=3$ Error bars \pm S.E.M). (C) Q-PCR showed up- regulation of lineage specific genes: $C d x 2$ (mesoderm), Pax6 (ectoderm) and Mixl (endoderm) in cells treated with RF embryoid bodies relative to respective iPSC line $(n=3$ Error bars \pm S.E.M). 
such as Cdx2 (mesoderm), Pax6 (ectoderm) and MixI (endoderm) by qRT-PCR analysis (Fig. 2C).

\section{Over-expression of Mbd3b enhances reprogramming of human fibroblasts}

We next looked at over-expression of $M b d 3 b$, a major isoform expressed in pluripotent stem cells $(8,11)$ and confirmed by immunoblotting an approximately $50 \%$ significant increase in the levels of Mbd3b (Fig. 3A). An almost two-fold significant $(\mathrm{p}<0.05)$ increase in the number of alkaline phosphatase colonies was observed in cultures transfected with $\mathrm{RF}+\mathrm{Mbd} 3 \mathrm{~b}$ when compared with $\mathrm{RF}$ alone (Fig. 3B) (BJ: RF 155 \pm 5 and RF+Mbd3b 290 \pm 10 ; RPD1: RF $30 \pm 1.5$ and $R F+M b d 3 b$ 64 \pm 1.3 ; PD1: RF
$70 \pm 2.0$ and $\mathrm{RF}+\mathrm{Mbd} 3 \mathrm{~b} 150 \pm 1.0$ ). iPSC colonies emerged at approximately the same time in cells treated with $\mathrm{RF}+\mathrm{Mbd} 3 \mathrm{~b}$ and in RF alone cultures and the overall proliferation kinetics of iPSC between these two conditions were similar (data not shown). iPSC clones from both these conditions expressed pluripotent markers (TRA-1-81 and TRA-1-60) with evidence of endogenous expression of Nanog, Oct 4 and Sox 2 by qRT-PCR (Fig. 3C, D) with no difference in level of expression. Importantly, iPSCs derived from fibroblasts transfected with $\mathrm{RF}+\mathrm{Mbd} 3 \mathrm{~b}$ formed EBs with comparable kinetics to that observed with cells treated RF alone (Fig. 3E) and expressed markers indicative of the three germ layers (Fig. 3F, G).
A

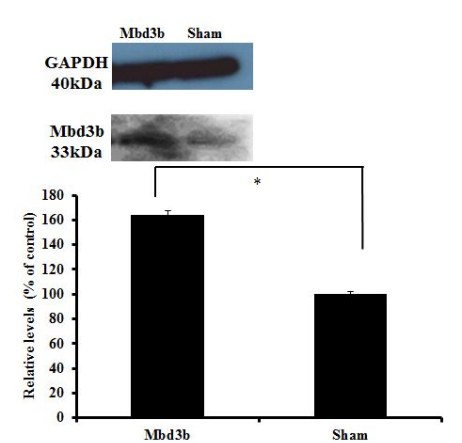

$\mathbf{C}$

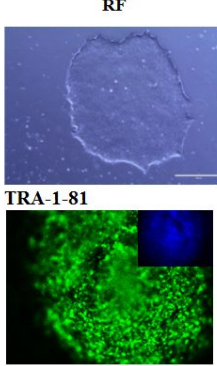

RF+Mbd3b

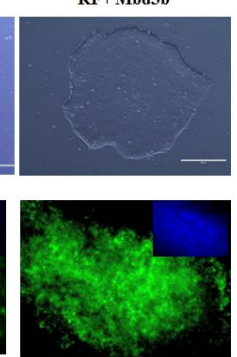

TRA-1-60

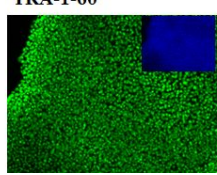

B

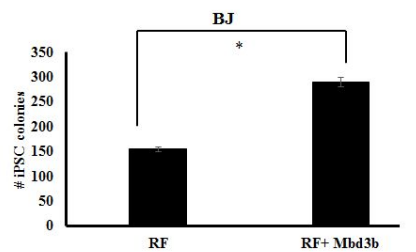

PD1

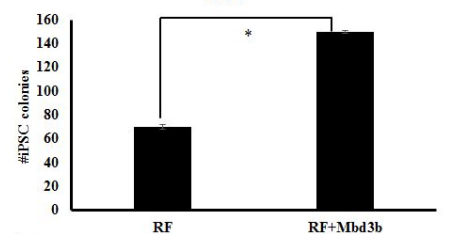

D

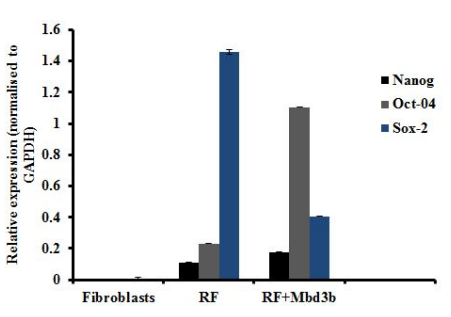

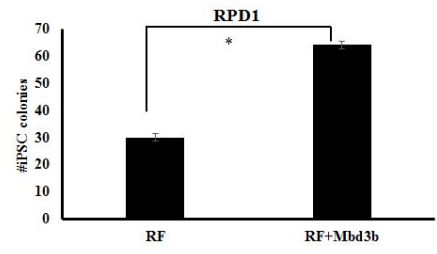

E $\quad$ RF $\quad \mathbf{R F}+\mathbf{M b d 3 b}$

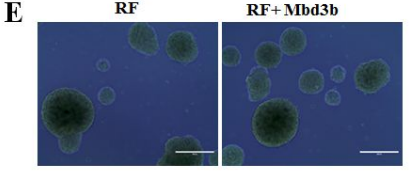

F

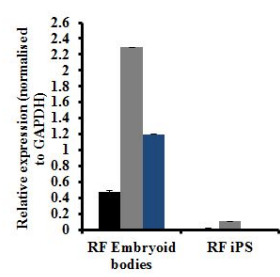

G

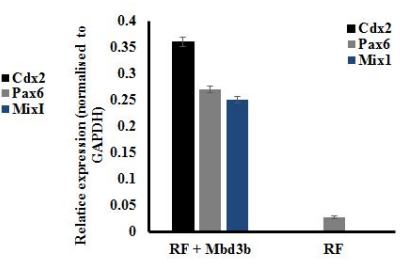

Fig. 3. Over-expression of Mbd3b facilitates reprogramming. (A) Immunoblot analysis for cells over-expressing Mbd3b compared to sham. Densitometry plot showed that cells over-expressing Mbd3b increased its expression by almost $50 \%$ compared to sham $(p<0.05)$. (B) Quantification of iPSC colonies generated from fibroblasts transfected with RF +Mbd3b compared to cells treated with RF in BJ neonatal cell line and patient fibroblasts (RPD1 and PD1). A two fold increase is seen in the number of colonies in RF + Mbd3b compared to RF only $(p<0.05)$. (C) Representative phase images of iPSC of established cell lines transfected with RF and RF + Mbd3b. Immunostaining with pluripotency markers (green) and counter staining with DAPI (blue) of cells treated with RF and RF + Mbd3b with TRA-1-81 and TRA-1-60. Scale bar is $200 \mu \mathrm{m}$. (Error bars \pm S.E.M). (D) Q-PCR showed expression of pluripotency genes (Oct-4, Nanog and Sox-2 respectively) in cells treated with RF and RF + Mbd3b. All results were normalised to GAPDH ( $n=3$ Error bars \pm S.E.M). (E) Representative images of embryoid bodies (EBs) generated from RF (left), and RF+Mbd3b (right). (F) Q-PCR showed up-regulation of lineage specific genes: Cdx2 (mesoderm), Pax6 (ectoderm) and Mixl (endoderm) in conditions treated with RF EBs and RF+Mbd3b EBs (G) relative to respective iPSC line. 


\section{Discussion}

Our study is the first to investigate the role of $M b d 3$ in the induction of pluripotency in patient derived primary fibroblasts, a convenient source of cells for iPSC cell-based therapies. Therefore, the identification of factors that enhance reprogramming efficiency of primary fibroblast derived lines will have direct clinical relevance (3). Our results show that partial knock-down of $M b d 3$ in human fibroblasts reduced the reprogramming efficiency. The iPSCs expanded from fibroblasts transfected with $\mathrm{RF}+\operatorname{shpMbd} 3$ displayed all the hallmarks of pluripotency suggesting that Mbd3 is not required for the establishment of pluripotency as reported previously $(7,8)$. However, the iPSCs expanded and derived from fibroblasts treated with $\mathrm{RF}+\operatorname{shpMbd} 3$ failed to form EBs confirming that $M b d 3$ is essential for forward differentiation into trilineage progenitors $(4,8)$.

In contrast, we found that over-expressing $M b d 3 b$ along with Yamanaka episomal plasmids enhances reprogramming efficiency by a two-fold. Whilst both forms of Mbd3 $(M b d 3 a$ and $M b d 3 b)$ interact with NuRD components, over-expression of $M b d 3 b$ has been shown to result in more pronounced phenotype than other isoforms in reversing the effects of knock-down of Mbd3 (11). Our results showed that over-expression of $M b d 3 b$ along with $\mathrm{RF}$ increased the number of iPSC cell colonies formed without affecting the attributes of pluripotency including the ability generate embryoid bodies with evidence of trilineage differentiation. The in-vitro differentiation potential in this study provides with robust reproducible alternative to teratoma assay and is sensitive enough to assess the pluripotency characteristics of iPSCs (15). However, further studies in a teratoma model are nevertheless required to establish this aspect in an unequivocal manner.

Our results reported herein are in agreement with reports by Onder et al.; 2012 (9) dos Santos et al.; 2014 (8, 9) and Zhang et al.; 2016 (16) but are at odds with two other reports $(6,7)$. In our opinion, the latter studies are not directly comparable as they used different methods of reprogramming and target cell lines. However, future studies are required to show the effect of $M b d 3$ using retrovirus or lentiviral method of reprogramming fibroblasts. Interestingly, others have shown that the Mbd3-miR-134 axis plays a key role in induction of pluripotency with Mbd3 overcoming the repression of reprogramming caused by over-expression of miR-134 (16). How this happens is under investigation with recent data showing an interdependence among $\mathrm{Mbd} 3 / \mathrm{NuRD}$, Tet1 and $5 \mathrm{hmC}$ to maintain normal chromatin structure and gene regulation in pluripotent stem cells (17).

We, therefore, conclude that $M b d 3$ plays an important role in reprogramming of human fibroblast cells using the episomal plasmid-based system of reprogramming. Understanding detailed molecular mechanism of $M b d 3$ in reprogramming and their function in enhancing the reprogramming efficiency are worthy of further investigation, and will be helpful in deriving excellent quality of iPSCs to facilitate clinical application.

\section{Acknowledgments}

This work was supported by Medical Research Council MR/L022842/1. The funders had no role in the study design, data collection and analysis, decision to publish, or preparation of the manuscript.

\section{Potential Conflict of Interest}

The authors have no conflicting financial interest.

\section{Supplementary Materials}

Supplementary data including supplementary methods can be found with this article online at http://pdf.medrang. co.kr/paper/pdf/IJSC/IJSC-11-s18036.pdf.

\section{References}

1. Takahashi K, Tanabe K, Ohnuki M, Narita M, Ichisaka T, Tomoda K, Yamanaka S. Induction of pluripotent stem cells from adult human fibroblasts by defined factors. Cell 2007;131:861-872

2. Okita K, Matsumura Y, Sato Y, Okada A, Morizane A, Okamoto S, Hong H, Nakagawa M, Tanabe K, Tezuka K, Shibata T, Kunisada T, Takahashi M, Takahashi J, Saji H, Yamanaka S. A more efficient method to generate integration-free human iPS cells. Nat Methods 2011;8:409-412

3. Goh PA, Caxaria S, Casper C, Rosales C, Warner TT, Coffey PJ, Nathwani AC. A systematic evaluation of integration free reprogramming methods for deriving clinically relevant patient specific induced pluripotent stem (iPS) cells. PLoS One 2013;8:e81622

4. Kaji K, Nichols J, Hendrich B. Mbd3, a component of the NuRD co-repressor complex, is required for development of pluripotent cells. Development 2007;134:1123-1132

5. Hendrich B, Guy J, Ramsahoye B, Wilson VA, Bird A. Closely related proteins MBD2 and MBD3 play distinctive but interacting roles in mouse development. Genes Dev 2001;15:710-723

6. Luo M, Ling T, Xie W, Sun H, Zhou Y, Zhu Q, Shen M, Zong L, Lyu G, Zhao Y, Ye T, Gu J, Tao W, Lu Z, Grummt I. NuRD blocks reprogramming of mouse somatic cells into pluripotent stem cells. Stem Cells 2013;31:1278-1286

7. Rais Y, Zviran A, Geula S, Gafni O, Chomsky E, Viukov 
S, Mansour AA, Caspi I, Krupalnik V, Zerbib M, Maza I, Mor N, Baran D, Weinberger L, Jaitin DA, Lara-Astiaso D, Blecher-Gonen R, Shipony Z, Mukamel Z, Hagai T, Gilad S, Amann-Zalcenstein D, Tanay A, Amit I, Novershtern N, Hanna JH. Deterministic direct reprogramming of somatic cells to pluripotency. Nature 2013;502:65-70

8. dos Santos RL, Tosti L, Radzisheuskaya A, Caballero IM, Kaji K, Hendrich B, Silva JC. MBD3/NuRD facilitates induction of pluripotency in a context-dependent manner. Cell Stem Cell 2014;15:102-110

9. Onder TT, Kara N, Cherry A, Sinha AU, Zhu N, Bernt KM, Cahan P, Marcarci BO, Unternaehrer J, Gupta PB, Lander ES, Armstrong SA, Daley GQ. Chromatin-modifying enzymes as modulators of reprogramming. Nature 2012; 483:598-602

10. Roloff TC, Ropers HH, Nuber UA. Comparative study of methyl-CpG-binding domain proteins. BMC Genomics 2003;4:1

11. Kaji K, Caballero IM, MacLeod R, Nichols J, Wilson VA, Hendrich B. The NuRD component Mbd3 is required for pluripotency of embryonic stem cells. Nat Cell Biol 2006;8: 285-292

12. Beers J, Gulbranson DR, George N, Siniscalchi LI, Jones J, Thomson JA, Chen G. Passaging and colony expansion of human pluripotent stem cells by enzyme-free dissociation in chemically defined culture conditions. Nat
Protoc 2012;7:2029-2040

13. McIntosh J, Lenting PJ, Rosales C, Lee D, Rabbanian S, Raj D, Patel N, Tuddenham EG, Christophe OD, McVey JH, Waddington S, Nienhuis AW, Gray JT, Fagone P, Mingozzi F, Zhou SZ, High KA, Cancio M, Ng CY, Zhou J, Morton CL, Davidoff AM, Nathwani AC. Therapeutic levels of FVIII following a single peripheral vein administration of rAAV vector encoding a novel human factor VIII variant. Blood 2013;121:3335-3344

14. Štefková K, Procházkováv J, Pacherník J. Alkaline phosphatase in stem cells. Stem cells Int 2015 doi: 10.1155/ 2015/628368

15. Buta C, David R, Dressel R, Emgård M, Fuchs C, Gross U, Healy L, Hescheler J, Kolar R, Martin U, Mikkers H, Müller FJ, Schneider RK, Seiler AE, Spielmann H, Weitzer G. Reconsidering pluripotency tests: do we still need teratoma assays? Stem Cell Res 2013;11:552-562

16. Zhang L, Zheng Y, Sun Y, Zhang Y, Yan J, Chen Z, Jiang H. MiR-134-Mbd3 axis regulates the induction of pluripotency. J Cell Mol Med 2016;20:1150-1158

17. Koh KP, Yabuuchi A, Rao S, Huang Y, Cunniff K, Nardone J, Laiho A, Tahiliani M, Sommer CA, Mostoslavsky G, Lahesmaa R, Orkin SH, Rodig SJ, Daley GQ, Rao A. Tet1 and Tet2 regulate 5-hydroxymethylcytosine production and cell lineage specification in mouse embryonic stem cells. Cell Stem Cell 2011;8:200-213 